\title{
PEDICULOSE NOS CENTROS DE EDUCAÇÃO INFANTIL: CONHECIMENTOS E PRÁTICAS DOS TRABALHADORES
}

\author{
Pediculosis in children attending day care centers: knowledge \\ and practice of workers \\ Infestión de piojos en los centros de educación infantil: \\ conocimientos y prácticas de los trabajadores
}

Flávia Lopes Gabani ${ }^{1}$

Clarice Martins Lima Maebara ${ }^{2}$

Rosângela Aparecida Pimenta Ferrari ${ }^{3}$

\begin{abstract}
RESUMO
Este trabalho objetivou identificar e analisar conhecimentos e práticas, acerca da pediculose, dos trabalhadores de Centros de Educação Infantil (CEI) das áreas de abrangência de duas Unidades de Saúde da Família, Londrina, Paraná. Trata-se de estudo descritivo transversal. Foi aplicado formulário com questões sobre mitos, tabus, práticas, dúvidas e dificuldades na interrupção da infestação da pediculose em oito CEl. Posteriormente, realizou-se oficina com materiais educativos. Participaram $60,4 \%$ dos funcionários, aqueles que estavam presentes no dia determinado pela instituição. Quase a totalidade referiu infestação pela ectoparasitose, assim como em seus familiares. A problemática é enfrentada cotidianamente nos CEl (72,1\%). Práticas adequadas de enfrentamento ainda são limitadas. Muitos mitos e tabus persistem (piolhos pulam, uso de sal, vinagre e querosene, etc.). As principais dúvidas estão relacionadas ao tratamento, e a maior dificuldade é a falta de colaboração dos pais. Ressalta-se, enfim, necessidade e importância de ações intersetoriais, preventivas e sistemáticas, no ambiente escolar.
\end{abstract}

Palavras-chave: Enfermagem Pediátrica. Piolhos. Creches. Cuidado da Criança.

\begin{abstract}
This study aimed to identify and analyze knowledge and practices, about pediculosis, from employees at Child Day Care Centers at two areas of coverage of the Family Health Units, Londrina, Paraná. It's descriptive transversal study. Form was applied with questions about myths, taboos, practices, doubts and difficulties in stopping the infestation of pediculosis in eight Child Day Care Centers. Subsequently, workshop was held with educational materials. $60.4 \%$ of employees participated, those who were present on the day determined by the institution. Almost all indicated the ectoparasitic infestation, as well as their families. The problem is faced daily in Child Day Care Centers (72.1\%). Practice appropriate care is still limited. Many myths and taboos persist (lice jump, use salt, vinegar and kerosene, etc). The main questions are related to treatment and the greatest difficulty is the lack of cooperation from parents. It emphasizes, finally, the need and the importance of internal actions, preventive and systematic in the school environment.
\end{abstract}

Keywords: Pediatric Nursing. Lice. Child day care centers. Child care.

\section{Resumen}

Este estudio tuvo como objetivo identificar y analizar los conocimientos y prácticas acerca de la pediculosis que tienen los empleados de los Centros de Educación Infantil (CEI), de las áreas que abarcan dos unidades de Salud de la Familia, Londrina, Paraná. Se trata de un estudio descriptivo transversal. Se aplicó un formulario con preguntas acerca de mitos y tabúes, prácticas, dudas y dificultades para poner fin a la infestación de piojos en ocho CEl. Posteriormente, se realizó un taller con materiales educativos. Participó el $60,4 \%$ de los empleados, los que estaban presentes en el día determinado por la institución. Casi todos reportaron la infestación parasitaria, así como en sus familias. El problema es enfrentado diariamente en los CEI (72,1\%). Prácticas adecuadas para hacer frente as este problema aún son limitadas. Persisten muchos mitos y tabúes (que los piojos saltan, el uso de la sal, del vinagre y de la parafina, etc.) Las principales dudas se relacionan con el tratamiento y la mayor dificultad que se presenta es la falta de cooperación de los padres. Por último, cabe señalar la necesidad y la importancia de que se implementen acciones intersectoriales, preventivas y sistemáticas en el ambiente escolar.

Palabras clave: Enfermería Pediátrica. Piojos. Jardines infantiles. Cuidado del niño.

\footnotetext{
'Enfermeira. Residente de Enfermagem em Saúde da Criança, nível especialização - Universidade Estadual de Londrina (UEL), Londrina, Paraná, Brasil. E-mail: flavialopes23@gmail.com,²Enfermeira. Residente de Enfermagem em Saúde da Criança, nível especialização - Universidade Estadual de Londrina (UEL), Londrina, Paraná, Brasil. E-mail: cacamaebara@gmail.com, ${ }^{3}$ Enfermeira. Professor Assistente Mestre do Departamento de Enfermagem - Área Saúde da Criança e do Adolescente. Coordenadora da Residência de Enfermagem em Saúde da Criança - Universidade Estadual de Londrina (UEL), Londrina, Paraná, Brasil. E-mail: ropimentaferrari@uel.br
} 


\section{INTRODUÇÃO}

Apesar dos avanços e investimentos voltados ao setor saúde no Brasil, discrepâncias socioeconômicas ainda estão presentes em todo território nacional. A despeito disso, muitas doenças caracterizadas como marcas de um país subdesenvolvido já não apresentam percentuais significativos em determinadas regiões. Outras, no entanto, encontram-se mais evidentes no público carente e podem levar a graves consequências se não prevenidas, como, por exemplo: verminoses, escabiose, tungíase, larva migrans e pediculose. ${ }^{1}$

Dentre essas se ressalta a pediculose visto que ao longo dos séculos continua sendo um problema de saúde pública, principalmente nos aglomerados populacionais, como nos Centros de Educação Infantil (CEI), pela inexistência de ferramentas sistemáticas instituídas para inter romper sua cadeia de infestação.

A pediculose é causada por ectoparasita hematófago (Pediculus humanus capitis) o qual desenvolve todo seu ciclo de vida no ser humano. Frequentemente surgem lesões pruriginosas no couro cabeludo, local mais afetado, levando a riscos potenciais de complicações, quais sejam: infecções bacterianas (por exemplo, impetigo), micoses e, até mesmo, miíases. $^{2-3}$ Apesar de essas infestações atingirem 0 homem há milhares de anos em todas as partes do mundo, sendo vistas em múmias egípcias de 3.000 anos a.C., em pentes da época de Cristo encontrados nos desertos de Israel e em múmias do Peru pré-colombiano, ainda não existem estudos que permitam compreender de forma ampla a epidemiologia dessa doença e os cuidados prestados por profissionais e familiares no combate desta ectoparasitose. ${ }^{4}$

Não obstante, instituições escolares não possuem normas padronizadas para manejo dessa ocorrência levando ao uso indiscriminado de produtos piolhicidas. Outrossim, alguns pais continuam não tomando providências diante do incômodo problema, sendo dessa forma delegadas às instituições de ensino. ${ }^{2-3}$

Para o crescimento e desenvolvimento saudável da população infantil, é essencial organizar e planejar ações educativas voltadas à promoção do cuidado em saúde. Contudo, uma das dificuldades enfrentadas no ambiente de trabalho dos educadores se refere à definição das responsabilidades, ou seja, quais são de competência da família, da instituição educacional ou do serviço de saúde. ${ }^{5}$

Neste contexto, os cuidados relacionados ao corpo ficam sob responsabilidade dos familiares, enquanto aqueles voltados ao desenvolvimento infantil, incluindo relacionamento interpessoal, preenchem as práticas educativas dos CEI. Sobrepondo essa divisão virtual há opiniões divergentes que geram conflitos entre as partes em questão, por exemplo, quanto à responsabilidade de remover os piolhos adquiridos no ambiente escolar. Dessa forma, muitas práticas são realizadas empiricamente, sem nenhum preparo profissional adequado, fato capaz de por em risco o crescimento e o desenvolvimento saudável das crianças. ${ }^{5}$

Quando a equipe de saúde atua nos CEI geralmente é de forma pontual e restrita, isto é, em campanhas de vacinação ou nos surtos de doenças infectocontagiosas. Sendo assim, a existência de práticas entre os setores saúde e educação para instituir promoção e prevenção entre escolares continua aquém das reais necessidades da população infantil e suas famílias. Se não há ações integrais e intersetoriais, resta aos educadores, de alguma forma, lidar com a questão pediculose, mesmo que ainda não estejam preparados para o enfrentamento da interrupção da cadeia de infestação.

Desta forma, a presente pesquisa justifica-se ao considerar a necessidade e importância da parceria e trabalho conjunto entre unidades locais de saúde e educação infantil para o combate à infestação entre os menores de seis anos. Para tanto, consideramos que, ao preparar os profissionais que prestam cuidados às crianças, em geral em período integral, poder-se-á intervir para redução da pediculose neste ambiente escolar e diminuir os riscos à saúde deste grupo etário.

Assim, o presente trabalho objetivou identificar e analisar conhecimentos e práticas, acerca da pediculose, dos trabalhadores de CEI das áreas de abrangência de duas Unidades de Saúde da Família (USF) em Londrina, Paraná.

\section{MÉTODO}

Trata-se de estudo descritivo transversal, no qual foram considerados os CEI das áreas de abrangência de duas USF do município de Londrina, Paraná, durante o primeiro semestre de 2008. 
A "USF A" possui população estimada em 9.701 pessoas, sendo 2.714 famílias cadastradas no Sistema de Informação de Atenção Básica (SIAB) e possui três Equipes de Saúde da Família (ESF). Sua área de abrangência é diversificada, sendo encontradas tanto famílias de melhor nível socioeconômico como moradores de áreas de risco. Crianças menores de cinco anos representam 1,7\% (167) dos integrantes das famílias cadastradas, 9,1\% (888) estão entre cinco e nove anos e 10,2\% (993) entre 10 e 14 anos. Nesta área há oito $\mathrm{CEI}$, sendo a grande maioria de caráter filantrópico. ${ }^{6}$

A "USF B" mostra características semelhantes à outra USF por terem delimitações limítrofes. Sua população está estimada em 9.232 pessoas, sendo 2.658 famílias cadastradas. Possui, também, três ESF e áreas com discrepâncias sociais e econômicas. Crianças menores de cinco anos representam 2,7\% (254) dos integrantes das famílias cadastradas, 7,9\% (727) estão entre cinco e nove anos e 9,0\% (829) entre 10 e 14 anos. Nesta área há apenas dois CEI, sendo estes de caráter público. ${ }^{6}$

Desta forma, considerou-se no presente estudo oito CEI, sendo seis da área de abrangência da USF A e dois da USF B, haja vista que dois foram excluídos por terem feito parte do pré-teste. A casuística foi composta por funcionários destes centros (educadoras, cozinheiras e auxiliares da limpeza) que atuam, direta ou indiretamente, com crianças de até seis anos de idade, independente do tempo de serviço e de sua função na instituição educacional.

A amostra da pesquisa seria composta pela totalidade dos trabalhadores, contudo foi considerado aqueles presentes no dia escolhido pelas CEl. Todos os participantes concordaram com a participação e assinaram o Termo de Consentimento Livre e Esclarecido. Cabe salientar que, em outro momento, houve resgate daqueles que não participaram do estudo, com utilização da mesma metodologia de abordagem, para homogeneização e difusão do conhecimento, no entanto sem inclusão na amostra.

Após a identificação da necessidade de trabalhar sobre a temática pediculose por meio de visita aos CEI, foi realizado contato prévio com os diretores para agendar dia e horário mais adequados para a participação dos profissionais na pesquisa e na oficina de educação em saúde relacionada ao tema.

Para obtenção dos dados foi utilizado um formulário, previamente testado, composto pelas variáveis: sexo, idade, escolaridade, número de filhos, tempo de prestação de serviço infantil, experiência prévia com a pediculose (pessoal e profissional), frequência do contato, conhecimentos, dúvidas, dificuldades e manejo da ectoparasitose. A aplicação do formulário foi de caráter individual, não sendo permitida qualquer interação entre os participantes durante seu preenchimento.

Após sua aplicação, realizou-se a oficina utilizando-se materiais educativos: fotos, reportagens que abordaram os riscos do tratamento e cartazes com desenhos explicativos sobre o ciclo de vida do piolho e suas características, propiciando a troca de experiências entre os membros, solucionando dúvidas e realizando correções de práticas prejudiciais à promoção da saúde infantil para auxiliar esses trabalhadores a contornar as dificuldades vivenciadas no ambiente de trabalho e familiar.

Os dados coletados foram processados eletronicamente por meio do programa de domínio público Epi Info, resultando em tabulação e análise das frequências, além do Excel for Windows para melhor aplicabilidade das variáveis e organização dos dados em tabelas e figuras.

Esta pesquisa seguiu as normas relativas à pesquisa envolvendo seres humanos da Resolução 196/ 96 do Conselho Nacional de Saúde, sendo aprovada pelo Comitê de Ética e Pesquisa da UEL, pelo parecer $n^{\circ}$ 024/08.

\section{RESULTADO}

De um total de 101 funcionários nos oito CEI, $61(60,4 \%)$ participaram do estudo por estarem presentes no dia e horário definidos pela instituição.

A Tabela 1 ilustra a distribuição dos funcionários de acordo com variáveis sociodemográficas. Quase a totalidade é do sexo feminino $(98,4 \%)$, na faixa etária entre 25 e 38 anos (44,3\%), com escolaridade superior a oito anos de estudo (96,7\%), com filhos (70,5\%) e com mais de cinco anos de trabalho voltado ao público infantil $(55,7 \%)$. 
Tabela 1 - Variáveis sociodemográficas dos funcionários dos CEl, Londrina, Paraná, 2008

\begin{tabular}{cccc}
\hline Variáveis & Categorias & $\boldsymbol{n}$ & $\boldsymbol{\%}$ \\
\hline \multirow{2}{*}{ Sexo } & Masculino & 1 & 1,6 \\
& Feminino & 60 & 98,4 \\
& $18 \vdash 25$ & 14 & 23,0 \\
Idade (anos) & $25 \vdash 39$ & 27 & 44,3 \\
& $\geq 39$ & 15 & 24,6 \\
& Ignorado & 5 & 8,1 \\
Escolaridade (anos) & $<8$ & 2 & 3,3 \\
& $>8$ & 59 & 96,7 \\
Filhos & Sim & 43 & 70,5 \\
& Não & 18 & 29,5 \\
& $<1$ & 5 & 8,2 \\
Tempo de trabalho (anos) & $1 \mathbf{r} 5$ & 22 & 36,1 \\
& $>5$ & 34 & 55,7 \\
\hline Total & & 61 & 100,0 \\
\hline
\end{tabular}

A experiência prévia com a pediculose está descrita na Tabela 2. Verifica-se que $72,1 \%$ dos funcionários já lidaram com a infestação desta ectoparasitose no ambiente familiar com seus filhos. Grande percentual dos profissionais já teve piolho quando criança e depois de adulto $(91,8 \%)$, sendo este último caso relacionado ao ambiente de trabalho, com frequência de uma a três vezes $(54,4 \%)$ até o momento da pesquisa, e 22,8\%, mais de seis vezes. Observa-se, também, que $72,1 \%$ enfrentam a problemática no cotidiano dos CEI, sendo $45,9 \%$ diariamente e $26,2 \%$ semanalmente, seguidos de $18,0 \%$ mensalmente.

Tabela 2 - Experiências dos funcionários dos CEI com a pediculose no núcleo familiar e no ambiente de trabalho, Londrina, Paraná, 2008

\begin{tabular}{llrr}
\hline Variáveis & Categorias & $\boldsymbol{n}$ & \% \\
\hline Seus filhos tiveram piolho* & Sim & 31 & 72,1 \\
& Não & 10 & 23,3 \\
& Ignorado & 2 & 4,7 \\
Você já teve piolho & Sim & 56 & 91,8 \\
& Não & 4 & 6,6 \\
& Ignorado & 1 & 1,6 \\
Quantas vezes você teve piolho** & 1 - 4 & 31 & 54,4 \\
& 4 - 6 & 10 & 17,5 \\
& $\geq 6$ & 13 & 22,8 \\
Frequência de piolhos no trabalho & Ignorado & 3 & 5,3 \\
& Diariamente & 28 & 45,9 \\
& Semanalmente & 16 & 26,2 \\
& Quinzenalmente & 2 & 3,3 \\
& Mensalmente & 11 & 18,0 \\
& Ignorado & 4 & 6,6 \\
\hline Total & & 61 & 100,0 \\
\hline${ }^{*} \mathrm{n}=43$. Excluídos aqueles que não têm filhos. & & \\
${ }^{* *} \mathrm{n}=57$. Excluídos aqueles que não tiveram piolho. & &
\end{tabular}


A Tabela 3 relaciona-se aos mitos e tabus acerca da pediculose; $80,3 \%$ dos funcionários acreditavam que piolhos provêm da falta de higiene e mais de 60,0\% entendiam que este ectoparasita pula e se prolifera mais frequentemente no verão. Quanto ao tratamento, o xampu comum foi considerado mais eficaz para a pediculose
$(42,6 \%)$, seguido pelo sal e vinagre $(31,1 \%)$. Chama a atenção que ainda $16,4 \%$ dos funcionários consideram "pesticidas" como forma de extermínio do piolho. Também consideram como verdadeiro que meninas têm mais piolhos que meninos $(36,1 \%)$.

Tabela 3 - Mitos e tabus dos funcionários dos CEl em relação à pediculose, Londrina, Paraná, 2008

\begin{tabular}{|c|c|c|c|c|}
\hline \multirow{3}{*}{ Mitos e Tabus } & \multicolumn{4}{|c|}{ Respostas } \\
\hline & \multicolumn{2}{|c|}{ Verdad eiro } & \multicolumn{2}{|c|}{ Falso } \\
\hline & $n$ & $\%$ & $n$ & $\%$ \\
\hline \multicolumn{5}{|l|}{ Meio de infestação do piolho } \\
\hline Piolhos voam & 22 & 36,1 & 39 & 63,9 \\
\hline Piolhos pulam & 40 & 65,6 & 21 & 34,4 \\
\hline Piolhos preferem o verão & 38 & 62,3 & 23 & 37,7 \\
\hline Piolhos provêm da falta de higiene & 49 & 80,3 & 12 & 19,7 \\
\hline Piolhos gostam de sangue doce & 11 & 18,0 & 50 & 82,0 \\
\hline \multicolumn{5}{|l|}{ Tratamento } \\
\hline Sal e vinagre matam piolhos & 19 & 31,1 & 42 & 68,9 \\
\hline Querosene e baygon matam piolhos & 10 & 16,4 & 51 & 83,6 \\
\hline Xampu comum pode matar piolhos & 26 & 42,6 & 35 & 57,4 \\
\hline \multicolumn{5}{|l|}{ Outras características } \\
\hline Cabelo curto e liso tem menos piolhos & 12 & 19,7 & 49 & 80,3 \\
\hline Men in as têm mais piolhos que meninos & 22 & 36,1 & 38 & 62,3 \\
\hline Quem tem caspa não tem piolhos & 4 & 6,6 & 57 & 93,4 \\
\hline Quem tem caspa tem mais piolhos & 7 & 11,5 & 54 & 88,5 \\
\hline
\end{tabular}

Dentre as práticas realizadas nos seus núcleos de convivência ( $n=99$ ), conforme ilustra a Figura 1, destacamse e catação e uso de pente fino $(42,4 \%)$ e uso de medicamentos $(34,4 \%)$, enquanto que pouco mais de $10 \%$ consideravam a educação dos familiares e crianças e melhora da higiene pessoal.

Figura 1 - Práticas dos funcionários dos CEI no combate à pediculose, Londrina, Paraná, 2008
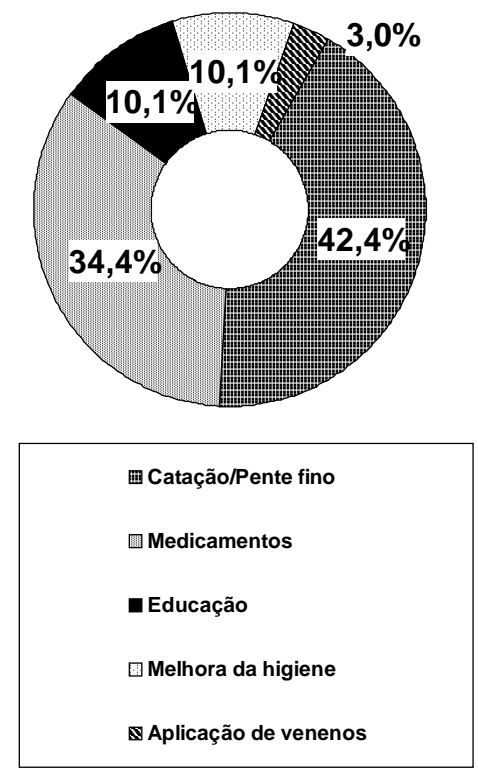

Das 58 dúvidas mais citadas sobre pediculose (Figura 2), ressalta-se aquelas referentes ao tratamento $(48,2 \%)$, seguidas do ciclo de vida e características peculiares do parasita $(25,9 \%)$.

Figura 2 - Dúvidas dos funcionários dos CEl acerca da pediculose, Londrina , Paraná, 2008

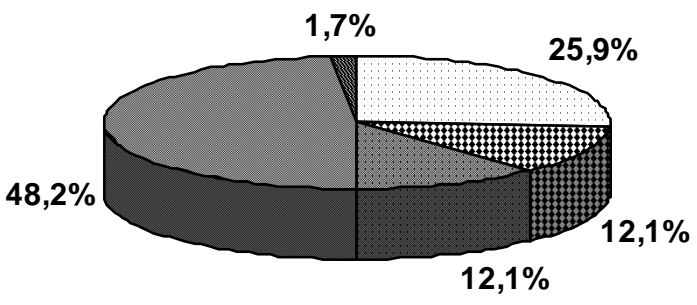

$\square$ Características do parasita

耳revenção

$\square$ Transmissão

$\square$ Tratamento

圈 Com plicações 
Com relação às dificuldades dos profissionais vivenciadas no ambiente de trabalho $(n=59)$, a falta de colaboração dos pais representou $81,3 \%$, além do ambiente de trabalho desfavorável, ou seja, aglomeração de crianças $(11,9 \%)$, desconhecimento do tratamento correto $(5,1 \%)$ e falta de apoio dos profissionais de saúde $(1,7 \%)$.

\section{DISCUSÃO}

O presente estudo procurou caracterizar os funcionários identificando variáveis sociodemográficas, além da experiência prévia com a pediculose. Infelizmente ainda não há pesquisas viáveis de comparação à temática. Como supracitado nos resultados, quase a totalidade dos funcionários dos CEI são mulheres, predominantemente jovens adultas e mães. Afirmaram ter tempo superior a cinco anos de trabalho na educação infantil. Experiências pessoais e profissionais relacionadas ao tema fazem parte do cotidiano doméstico e também de trabalho. Contudo, a forma correta de intervir na cadeia de infestação ainda é divergente.

Pesquisa realizada na província de La Rioja, Argentina, avaliou 1.370 crianças em idade escolar de 26 escolas. Verificouse que $22 \%$ apresentavam sinais de infestação recente, mas baixa probabilidade de parasitismo, porém $28 \%$ tinham sinais de infestação e pediculose ativa. Além disso, o estudo mostra que crianças de escolas públicas possuem maior prevalência da ectoparasitose se comparadas às de escolas particulares. ${ }^{7}$

Outras pesquisas nacionais convergem opiniões a respeito da maior proporção de infestação em crianças em relação aos adultos, principalmente oriundas de instituições escolares e durante o período letivo, reforçando a hipótese de que são os principais locais de transmissão pela aglomeração e características peculiares da população infantil. 8-10 $^{-10}$

Muitos mitos e tabus enraizados na sociedade dificultam o seu combate. Com relação ao comportamento desse ectoparasita, a maioria da população deste estudo referiu que muitos piolhos são capazes de voar e/ou pular, preferem o verão, provêm da falta de higiene e gostam de sangue doce.

0 piolho pertence à Ordem Phthiraptera, da Classe Insecta, são invariavelmente Ápteros ( $a=$ sem + pteros $=$ asas), ou seja, sua transmissão ocorre por contato, seja direto (pessoal) ou por uso de utensílios (boné, pente, travesseiro, presilha, etc.), e são incapazes de voar ou pular. Esses ectoparasitas se proliferam, principalmente, em ambientes quentes e úmidos, talvez daí a impressão de que preferem o verão. Porém as medidas preventivas devem ser mantidas durante todo o ano. Sua incidência não tem relação direta com a higiene, muito menos com barreiras sociais, econômicas ou geográficas, haja vista sua surpreendente infestação nos últimos anos, refletindo em grande aumento nas vendas de produtos piolhicidas. ${ }^{11}$ Infelizmente ainda faltam estudos referentes ao motivo da predisposição que algumas pessoas têm de adquirir mais piolhos que outras, contudo não há relação com o nível de açúcar no sangue. 2,4,12

As lêndeas são ovais, pequenas, de coloração brancoamarelada, hermeticamente fechadas e se fixam próximas ao couro cabeludo pela ação de um "cemento", ou seja, uma substância quitinosa de difícil remoção. Cabe salientar que não existem pesquisas que comprovem a ação do sal e do vinagre no combate ao piolho eà lêndea, porém alguns estudos afirmam que podem facilitar a dissolução dessa substância., 212 Fato, talvez, que comprove a resposta em nossa pesquisa de que esse método é eficaz na eliminação dessa ectoparasitose.

0 uso irracional e disseminado de produtos piolhicidas vem proporcionando grande resistência desses ectoparasitas, assim como as bactérias aos antimicrobianos, motivo pelo qual a indústria farmacêutica está sempre modificando e lançando novas fórmulas. Resistência é entendida como sobrevivência a concentrações ou doses de um produto que deveria causar mortalidade total. 0 xampu comum não possui essa propriedade, mas a população deste estudo consideram uma alternativa adequada para o tratamento da pediculose. ${ }^{11}$ Outro fato preocupante refere-se ao conceito de que querosene e baygon, extremamente tóxicos à população infantil, matam piolhos.

Outro mito encontrado é a afirmativa de que cabelo cur to e liso tem menos piolhos, visto que esse tipo de cabelo permite fácil visualização e "catação" dos ectoparasitas. Este conceito se expande aos que acham que meninas têm mais piolhos que meninos. ${ }^{2,4,12}$ Outro aspecto é que não existem estudos que comprovem, favoravelmente ou não, a existência de relação entre caspa e pediculose.

É mister salientar que muitos são os agravos predominantes entre a população infantil, seja de natureza ectoparasitária (pediculose, verminose, etc.) ou infecciosa transmissíveis (infecções respiratórias agudas, infecções urinárias, etc.). Cabe aos profissionais de saúde promover estratégias que previnam complicações dessas doenças, além da detecção precoce e ações articuladas de educação permanente para os núcleos de trabalho infantil e familiar.

Dentre os meios de tratamento, não há dúvida que a forma mecânica preventiva é a mais eficaz, ou seja, "catação" manual com uso de pente fino. ${ }^{2,4,12}$ No presente estudo, este método foi muito cotado, contudo muito próximo do uso de medicamentos, muitas vezes irracional, para controle de infestações. 
0 surgimento de produtos piolhicidas com promessas milagrosas, associado à globalização e amplas jornadas de trabalho dos familiares responsáveis, principalmente das mães, deixou a forma mecânica no esquecimento. Sua retomada é essencial ao combate à pediculose. Processos educativos para reciclagem conceitual devem alcançar não apenas educadores e escolares, mas todos os familiares envolvidos neste contexto, pois muitas são as dúvidas observadas com relação à característica do parasita, prevenção, transmissão, tratamento e complicações. Além disso, a conscientização é essencial, incluindo aqui os profissionais da saúde, para amenizar as dificuldades desses trabalhadores, proporcionando colaboração mútua entre os envolvidos na promoção do bem-estar infantil.

Um estudo para analisar a percepção das mães e educadoras diante dos cuidados oferecidos pelas creches do município de São Paulo concluiu que não há articulação entre os CEI e os serviços de saúde, sendo que, quando há aproximações, são de nível local, por questões circunstanciais, e não pelo estabelecimento de relações formais entre os níveis gerenciais. ${ }^{13}$

Nesse sentido, outros estudos vêm apontando, ao longo das décadas, a real necessidade de atividades de atualização para educadores, incluindo promoção da saúde infantil com manejos adequados aos agravos mais comuns da infância, como estratégia de aproximação dos serviços de saúde aos educacionais. ${ }^{14}$ Por outro lado, ficou expresso, na presente pesquisa, que a falta de colaboração dos pais e 0 ambiente de trabalho desfavorável estão entre as principais dificuldades no manejo dessa ectoparasitose; contudo, o desconhecimento do tratamento correto e a falta de apoio dos profissionais de saúde corroboram os achados supracitados.

Uma pesquisa realizada em creche no município do Rio de Janeiro mostra a importância do apoio do profissional da saúde, principalmente a equipe de enfermagem, na atuação direta frente aos educadores infantis. Assim como em nosso estudo, a pediculose foi vista entre os problemas de maior incidência em crianças de zero a quatro anos, reafirmando a necessidade de apoio mútuo entre essas categorias profissionais, juntamente com o núcleo familiar, na promoção do bem-estar infantil. ${ }^{15}$

A agenda de compromissos para promoção da saúde integral da criança e redução da mor talidade infantil apresenta como desafio a conformação de uma rede única integrada de assistência à criança com ocorrência de estratégias e ações prioritárias que envolvam esses serviços de apoio, conforme suas demandas e especificidades, para o cumprimento dos objetivos propostos pelos seus princípios norteadores. Sendo assim, o Ministério da Saúde aponta como primeiro princípio norteador o planejamento e desenvolvimento de ações intersetoriais, incluindo interfaces entre os serviços locais de saúde com a educação infantil, isto é, pré-escolas, contemplando parcerias e trabalhos articulados, haja vista que esse ambiente se mostra adequado para que ocorram de forma efetiva as práticas de educação em saúde entre escolares, familiares, funcionários e profissionais da saúde. ${ }^{16}$

De acordo com o Ministério da Saúde, ${ }^{17}$ conceitua-se como educação permanente em saúde ações educativas embasadas na problematização do processo de trabalho em saúde e que tenham como objetivo a transformação das práticas profissionais e da própria organização do trabalho, tomando como referência as necessidades de saúde das pessoas e das populações, a reorganização da gestão setorial e a ampliação dos laços da formação com o exercício do controle social em saúde. Sendo assim, esse processo educacional passa a ser pivô da relação ensino-serviço-comunidade, proporcionando momentos de mudança conceitual com reflexão das práticas consideradas inerentes ao bem-estar infantil.

Para efeito comparativo, um estudo que relacionou conhecimentos e práticas de educadoras de creche, porém relativos à anemia, concluiu que a grande maioria partia do senso comum, associado às suas próprias concepções de mundo, costumes, valores e crenças, e sedimentos nas experiências vivenciadas. Muitas apresentavam conceitos errôneos e prejudiciais à prestação de cuidados e expunham dificuldades na construção de parcerias entre educadores, familiares e profissionais de saúde, afetando de modo global o bem-estar infantil. ${ }^{18}$ No presente estudo também os mitos e tabus estiveram fortemente presentes quanto à forma de lidar com a pediculose, inclusive naqueles que vivenciam no ambiente familiar.

Outra pesquisa analisou os conhecimentos e práticas de trabalhadoras de creche relativos aos agravos respiratórios na infância e considerou que os mesmos são apropriados, porém as educadoras manifestam a necessidade de atualizações constantes no desejo de agirem com base científica, saindo do senso comum. Ressaltam que para adquirem esses conhecimentos é imprescindível orientação contínua dos profissionais da saúde por meio de encontros presenciais para discussão de temas relacionados. Dentre as dificuldades encontradas destaca-se, assim como neste estudo, a falta de comunicação entre educadores e familiares. ${ }^{19}$

Conhecimentos e práticas corretas e atualizadas do cuidado à infância refletem a qualidade do atendimento infantil, seja na prevenção da doença, promoção ou reabilitação da saúde. A união de esforços vai ao encontro das metas para redução da morbidade infantil. Educadores são potentes aliados 
das unidades de saúde, atuando como multiplicadores do conhecimento científico no combate a mitos de diversos agravos, incluindo a pediculose, capazes de pôr em risco desenvolvimento e crescimento saudável das crianças. Atuam, no cotidiano de seu trabalho, como ponte entre os profissionais da saúde e familiares, tendo como escopo a formação da consciência em luta da cidadania e bem-estar coletivo, ou seja, das crianças.

\section{CONSIDERAÇÕES FINAIS}

Enfim, a pediculose é vista como uma ectoparasitose prevalente entre a população infantil e de difícil resolutividade. Apesar de os funcionários dos CEl em questão vivenciarem frequentemente a presença de infestação, seja em seus núcleos familiares ou profissionais, muitas de suas práticas ainda se embasam no senso comum, sem conhecimento e respaldo científico. 0 uso de medicamentos é feito, muitas vezes, empiricamente e sem orientação médica, além disso, é considerado como verdade o uso de venenos agressivos e mortais às crianças para seu combate. A "catação" e a "penteação" ainda são muito usadas e eficazes, mas por meio de técnicas errôneas e arriscadas. Existem muitas dúvidas e dificuldades relacionadas à prevenção e tratamento, corroborando a necessidade de atenção especial ao tema abordado.

Cabe salientar que a oficina foi uma primeira aproximação com intuito de respaldar os trabalhadores, sendo ainda necessário o fortalecimento das relações entre USF e CEI para amplificação e incentivo de trabalho sistemático de prevenção e promoção da saúde infantil. Nesta perspectiva, acrescenta-se a necessidade de novas pesquisas para avaliar o impacto da educação em saúde realizada com esses profissionais.

\section{AGRADECIMENTOS}

Agradecemos a colaboração das coordenadoras e trabalhadores dos CEI durante o estudo, bem como das enfermeiras das USF e das Residentes de Enfermagem em Saúde da Criança Marcela de Oliveira Demitto e Nataly Tsumura Inocencio Soares pela importante parceria neste trabalho.

\section{REFERÊNCIAS}

1. Heukelbach J, Oliveira FAS, Feldmeier H. Ectoparasitoses e saúde pública no Brasil: desafios para controle. Cad Saude Publica. 2003 set/out; 19(5): 1535-540.
2.Andrade CF, Santos LU, Cecílio ATB.. Controle da pediculose: um projeto educativo. Manual do professor [citado 08 set 2008]. Disponivel em: http://www.piolho.org.br/artigos/apostila.pdf.

3.Catalá S, Carrizo L, Córdoba M, Khairallah R, Moschella F, Bocca JN, et al. Prevalência e intensidade da infestação por pediculus humanus capitis em escolares de seis a onze anos. Rev Soc Bras Med Trop 2004 nov/dez; 37(6): 499-501.

4.Portal do Piolho. História no mundo [citado 2008 set 10]. Disponível em: http://www.piolho.org.br/piolho.html.

5.Maranhão DG. 0 cuidado como elo entre saúde e educação. Cad Pesqu 2000 dez; 111: 115-33.

6.Ministério da Saúde (BR). DATASUS. Sistema de Informação da Atenção Básica -SIAB [citado 12 set 2008]. Disponível em: http:// www.datasus.gov.br/siab/siab.htm.

7.CataláS, Junco L, Vaporaky R. Pediculus capitis infestation according to sex and social factors in Argentina. Rev Saude Publica 2005 jun; 39(3): 438-43.

8.Linardi PM, De Maria M, Botelho JR, Hosken Cl, Cunha HC. Alguns fatores epidemiológicos relativos à infestação humana por pediculus capitis (Anoplura, Pediculidae) em Belo Horizonte, Minas Gerais, Brasil. Rev Bras Entomol 1995; 39: 921-29.

9.Borges R, Silva JJ, Rodrigues RM, Mendes J. Prevalence and monthly distribution of head lice using two diagnostic procedures in several age groups in Uberlândia, State of Minas Gerais, Southeastern Brazil. Rev Soc Bras Med Trop 2007 mar/abr; 40(2): 247-49.

10.Borges R, Mendes J. Epidemiological aspects of head lice in children attending day care centres, urban and rural schools in Uberlândia, Central Brazil. Memórias do Instituto Oswaldo Cruz 2002 mar; 97: 189-92.

11.Andrade CFS, Santos LU. Resistência dos piolhos aos inseticidas químicos - mecanismos, deteç̧ão, monitoramento e manejo [citado 15 set 2008]. Disponível em: http://www.piolho.org.br/artigos/ relato4.pdf .

12.Linardi PM. Anoplura. In: Melo AL, Linardi PM, Genaro 0. Parasitologia humana. São Paulo (SP): Atheneu; 2002. p. 368-72.

13.Bógus CM, Nogueira-Martins MCF, Moraes DEB, Taddei JAAC. Cuidados oferecidos pelas creches: percepções de mães e educadoras. Rev Nutr 2007 set/out; 20(5): 499-514.

14.Mohr A, Schall VT. Rumos da educação em saúde no Brasil e sua relação com a educação ambiental. Cad Saude Publica 1992 abr/jun; 8(2): 199-203.

15.Xavier TJS, Pinto FF, Souza MHN, Zeitoune RCG. Condições de saúde de crianças de creche comunitária e a enfermagem. Esc Anna Nery Rev Enferm 2003 ago; 7(2): 204-10.

16.Ministério da Saúde (BR). Secretaria de Atenção à Saúde. Departamento de Ações Programáticas Estratégicas. Agenda de compromissos para a saúde integral da criança e redução da mortalidade infantil. Brasília (DF); 2004. 
17.Ministério da Saúde (BR). Biblioteca Virtual em Saúde. Glossário Eletrônico [citado 25 set 2008]. Disponível em: http:// bvsms2.saude.gov.br/php/level.php?lang=pt\&component=44\&item $=$ 114.

18. Teixeira-Palombo CN, Fujimori E. Conhecimentos e práticas de educadoras infantis sobre anemia. Rev Bras Saude Matern Infant 2006 abr/jun; 6(2): 209-16.

19.Alves RCP, Veríssimo MDLOR. Conhecimentos e práticas de trabalhadoras de creches universitárias relativos às infecções respiratórias agudas na infância. Rev Esc Enferm USP 2006 mar; 40(1): 78-85.

\section{NOTA}

a Artigo extraído da monografia do curso de Especialização de Enfermagem em Saúde da Criança, Modalidade Residência. Departamento de Enfermagem do Centro de Ciências da Saúde (CCS), Universidade Estadual de Londrina (UEL). 